\title{
La competencia digital en estudiantes de magisterio. Análisis competencial y percepción personal del futuro maestro
}

\section{Digital competence in students of educational degrees. Analysis of future teachers' competence and perception}

\author{
Vicente Gabarda Méndez ${ }^{1}$ \\ vgabarda@campusviu.es \\ Ana Rodríguez Martín \\ ana.rodriguez@campusviu.es \\ María Dolores Moreno Rodríguez \\ mariadolores.moreno@campusviu.es \\ Universidad Internacional de Valencia, España
}

\begin{abstract}
Resumen:
La competencia digital del ciudadano se ha convertido en un elemento clave en las políticas educativas de cualquier etapa formativa. En este sentido, que el profesorado que imparte docencia en las diferentes enseñanzas haya adquirido y desarrollado sus propias destrezas en este ámbito, se postula como un prerrequisito básico para que los estudiantes puedan adquirir también esta competencia. En este trabajo se analiza la integración normativa de las Tecnologías de la Información y la Comunicación en las etapas de Educación Infantil y Educación Primaria, así como el planteamiento que se realiza de la formación tecnológica del profesorado como base para el estudio posterior. El
\end{abstract}

\begin{abstract}
:
Educational policies have highlighted digital competence as a key for citizens at any formative stage. In this way, teachers must have developed their own digital skills to be able to contribute to the student's development of their digital competence. In this study, we aimed to analyze the integration of Information and Communication Technologies within Early Childhood and Primary Education degrees and the technological training which teachers had received. The main objective of this study, based on the available policies, is to explore the perception that the students of Educational Degrees have of their own digital competence, using as a reference the Digital Competence Framework for Educa-
\end{abstract}

1 Dirección para correspondencia (correspondence address):

Vicente Gabarda Méndez. Área de Educación. Universidad Internacional de Valencia.

C/ Gorgos 5. 46021 Valencia (España). 
objetivo principal de este trabajo, partiendo de la regulación normativa, es explorar la percepción que tienen los estudiantes de los títulos de Magisterio acerca de su propia competencia digital, interpretando los resultados en base a la propuesta del Marco Común de Competencia Digital Docente del Ministerio de Educación, Cultura y Deporte. Para ello, se ha diseñado un cuestionario ad-hoc, que ha sido respondido por 104 estudiantes de los Grados de Educación Infantil y Educación Primaria. Los resultados evidencian que los estudiantes (y futuros docentes) tienen, de modo global, un nivel de competencia digital intermedio, obteniendo mayores puntuaciones en las áreas de información y comunicación y mayores carencias en las áreas de seguridad, resolución de problemas y creación de contenidos. Es importante resaltar, asimismo que hay divergencias entre la percepción personal de los futuros maestros sobre su competencia digital y las capacidades reales que demuestran los resultados.

\section{Palabras clave:}

Competencia; Curriculum de formación de profesorado; Utilización de la Tecnología en la Educación; Educación Infantil; Educación Primaria; Educación Superior. tors published by the Spanish Ministry of Education. We have designed a questionnaire, which has been answered by 104 students from the Early Childhood Education Degree and the Primary Education Degree. Results show that students (and future teachers) have, in general terms, an intermediate level of digital competence, showing good marks in the areas of Information and Communication. The lowest rates are linked to safety, problem solving and creation of technological contents. It is important to highlight that there are differences between the personal perception of the future teachers about their own digital competence and the real abilities that the marks show.

\section{Key words:}

Competence; teacher education curriculum; technology uses in education; early childhood education; Primary Education; Higher Education.

\section{Résumé:}

La compétence numérique du citoyen s'est convertie en élément clef des politiques éducatives de n'importe quelle étape formative. En ce sens, que le professorat qui accorde un enseignement dans différentes matières a acquis et développé sa propre compétence dans ce domaine. Cela est fortement recommandé comme condition basique, pour que les étudiants puissent aussi acquérir cette compétence. Dans ce travail, deux éléments sont analysés. D'une part, I'intégration normative des Technologies de I'Information et de la Communication, dans les étapes d'Education maternelle (infantil) et $d^{\prime}$ Enseignement Primaire. Et d'autre part, une mise en question quant à la formation technologique du professorat comme base pour l'étude postérieure. L'objectif principal, en partant de la régulation normative, est d'explorer la perception que les étudiants en Master ont à propos de leur propre compétence digitale, en interprétant les résultats sur la base de la proposition du Marco Común de Competencia Digital Docente du Ministerio de Educación, Cultura y Deporte. Pour cela, un questionnaire ad hoc a été réalisé, auquel 104 étudiants de licence d'Education maternelle (infantil) et d'Enseignement Primaire ont répondu. Les résultats mettent en évidence le fait que les étudiants (et futurs enseignants) ont un niveau intermédiaire en compétences digitales. Ils obtiennent des meilleurs résultats dans les domaines d'information et de communication, et éprouvent de plus grandes lacunes dans les domaines de la sécurité, de la résolution de problèmes et de la création de contenus. Il est donc important de mette cela en évidence, de la même manière qu'il y a des divergences entre la perception personnelle 
du futur enseignant sur sa compétence digitale, et les capacités réelles que démontrent les résultats.

\section{Mots clés:}

Compétence; Curriculum de formation de professorat; Utilisation de la Technologie dans I'Éducation; Éducation maternelle (infantil); Enseignement primaire; Education Supérieure.

Fecha de recepción: 12-5-2016

Fecha de aceptación: 24-2-2017

\section{Introducción}

\section{La regulación normativa de la competencia digital}

La competencia digital se ha convertido, en las últimas décadas, en un eje trasversal en la formación de cualquier ciudadano. Así se recoge en el documento de Competencias Clave para el Aprendizaje Permanente (Comunidades Europeas, 2007).

La asunción de esta competencia como elemento clave es el reflejo de la incorporación progresiva de las Tecnologías de la Información y la Comunicación (en adelante, TIC) en todas las esferas de la vida del ser humano, teniendo un impacto importante en sus ámbitos económico, social y personal.

En nuestro país, se han venido desarrollando iniciativas para la incorporación de las TIC en todas estas áreas, aunque se centre este estudio en el ámbito educativo. Este análisis pone el foco especialmente en cómo se ha planteado la integración de las TIC como elemento trasversal y qué alusiones se realizan a la formación tecnológica del profesorado.

Las primeras menciones directas a las tecnologías en la normativa educativa española se recogen con la entrada en vigor de la Ley Orgánica General del Sistema Educativo (LOGSE), de 3 de octubre de 1990, donde se recogía la necesidad de que las Administraciones Educativas tuvieran en cuenta los aspectos tecnológicos para la elaboración de los proyectos escolares. Las normativas posteriores (como la Ley Orgánica de Calidad Educativa, 2002) continúa con la propuesta de la LOGSE, introduciendo el término Tecnologías de la Información y tomando la formación del profesorado en su uso y la producción de materiales tecnológicos como un elemento clave para su introducción en las aulas.

Posteriormente, la Ley Orgánica de Educación, LOE (2006) da un 
paso más en la integración de las TIC en el sistema educativo. Recoge en su Preámbulo que, siguiendo las directrices europeas, es necesario mejorar la calidad y la eficacia de los sistemas de educación y de formación. Para ello, es necesario mejorar la capacitación de los docentes, de manera que puedan desarrollar las aptitudes necesarias en la sociedad del conocimiento y tener acceso a las TIC.

Específicamente, con respecto a la formación del profesorado en materia tecnológica, la LOE recoge, en su artículo 157f, dedicado a los Recursos para la mejora de los aprendizajes y apoyo al profesorado, que es necesario establecer programas de refuerzo para el aprendizaje de las tecnologías.

Por último, la Ley Orgánica para la Mejora de la Calidad Educativa (LOMCE, 2013), respeta de forma general los planteamientos de la LOE en relación a las tecnologías en sus artículos 111, 112 y 157, que regula la integración de las Tecnologías de la Información y la Comunicación. Asimismo, se expone en el Preámbulo que éstas se conciben como una herramienta clave en la formación del profesorado.

Al margen de estos planteamientos, es destacable que, tanto la LOE como la LOMCE proponen la integración curricular de las TIC como un elemento trasversal en todas las etapas educativas.

Así por ejemplo, la Ley Orgánica de Educación (2006) exponía en su artículo 17 que el estudiante debía iniciarse en la utilización de las tecnologías de la información y la comunicación, ligada al desarrollo de un espíritu crítico. Del mismo modo, en la Educación Infantil, se reconoce como principio pedagógico (artículo 14), que se debe fomentar una primera aproximación a las tecnologías de la información y la comunicación.

Estos mismos planteamientos se mantienen como principios básicos en la Ley Orgánica 8/2013, de 9 de diciembre, para la Mejora de la Calidad Educativa (2013).

Este interés sigue siendo explícito, posteriormente, en las normativas que regulan cada una de ellas (Real Decreto 1630/2006 de 29 de Diciembre sobre Enseñanzas mínimas del segundo ciclo de Educación Infantil y el Real Decreto 126/2014, de 28 de febrero, por el que se establece el currículo básico de la Educación Primaria), donde se reconoce de manera más explícita el papel de las TIC como elemento trasversal y de la competencia digital como requisito clave y equiparable a la competencia lingüística, matemática o social y ciudadana. 
Partiendo entonces de la premisa de que los estudiantes, al finalizar su escolaridad, deben haber desarrollado su competencia digital, resulta imprescindible que el profesorado que imparte docencia en estas etapas haya adquirido y desarrollado sus propias destrezas.

Si se analiza la normativa que regula los planes de formación inicial del profesorado de Educación Infantil y Primaria, se puede concluir que también se ha trasladado al ámbito de formación del profesorado la preocupación por la competencia digital.

En la Orden ECI 3854/2007, que regula la verificación de los títulos de Maestro/a en Educación Infantil, expone en su apartado 3 que uno de los objetivos primordiales en la formación del maestro de esta etapa es conocer las implicaciones educativas de las TIC, especialmente de la televisión.

En el caso de la Educación Primaria, la Orden ECI 3857/2007, que regula la verificación de los títulos de Maestro/a en Educación Primaria, igualmente se recoge como objetivo ya no solamente conocer las Tecnologías, sino también aplicarlas y discernir la información que efectivamente contribuya al aprendizaje (apartado 3).

Aunque a priori, se observa que efectivamente se recoge la formación tecnológica del profesorado de estas etapas como uno de los objetivos primordiales, no siempre se materializa este interés en los planes de estudio.

En este sentido, tal y como recogen Herrada y Herrada (2011), con la implantación del Espacio Europeo de Educación Superior, los planes de estudio de los Grados de Infantil y Primaria incluyen un menor número de asignaturas de carácter obligatorio que aborden contenidos TIC obstaculizando así la adquisición de estas competencias.

Partiendo de esta realidad, compleja y en ocasiones contradictoria, la propuesta de un marco que pueda determinar cuáles son las competencias y destrezas específicas que ha de adquirir y desarrollar un docente en materia tecnológica se convierte en un elemento de esencial valor.

\section{El Marco Común de Competencia Digital Docente frente a otros estándares}

Habiendo confirmado que la formación del profesorado es una pieza clave del desarrollo de la competencia digital de los estudiantes, se presenta el hándicap de identificar cuáles son específicamente las compe- 
tencias que debe adquirir éste para que el proceso se lleve a cabo con coherencia.

Ha habido, en los últimos años, varias propuestas por parte de organismos nacionales y supranacionales que han tratado de dar respuesta a este tema.

La International Society for Technology in Education (ISTE), propuso en 2008 los Estándares NETS para docentes, un marco donde se recogían algunos aspectos que se consideraban básicos para aprender y vivir de forma efectiva y productiva en el mundo digital. Se trataba de una propuesta basada en seis aspectos fundamentales: 1. El fomento del aprendizaje y la creatividad de los estudiantes; 2 . El diseño y desarrollo de aprendizaje y evaluaciones propias de la Era Digital; 3) El modelado del Trabajo y el Aprendizaje característicos de la Era Digital; 4) La promoción y ejemplificación de la Ciudadanía Digital y Responsabilidad; 5) El compromiso con el Crecimiento Profesional y con el Liderazgo.

Como puede comprobarse, la propuesta era vaga en cuanto al nivel de especificación de las destrezas docentes y se centraba más en el impacto que tendría la labor docente en el desarrollo de la competencia digital de sus estudiantes.

En una propuesta más reciente, y con una perspectiva más amplia, la Orientación Normativa Estratégica de la Alfabetización Mediática e Informacional de la Organización de Naciones Unidas (Organización de las Naciones Unidas para la Educación, la Ciencia y la Cultura, 2014) se orientaba a tres líneas estratégicas: el establecimiento de un marco de referencia común para el desarrollo de estrategias nacionales en esta materia, la facilitación de políticas que regulen los contenidos de los medios de comunicación y la elaboración y puesta a disposición una propuesta curricular ya desarrollada para la comunidad educativa.

Como parte de esta iniciativa, se establecen las directrices de la Alfabetización Mediática e Informacional. Currículum para profesores, identificando como aspectos clave para esta capacitación cuestiones como: 1) Entender el papel de los medios y de la información en la democracia; 2) Comprender el Contenido de los Medios y sus Usos; 3) Acceder a la Información de una manera Eficaz y Eficiente; 4) Evaluar Críticamente la Información y las Fuentes de Información; 5) Aplicar los Formatos Nuevos y Tradicionales en los Medios; 6) Situar el Contexto Sociocultural del Contenido de los Medios. En este caso, se trata de una propuesta vinculada más a destrezas mediáticas, ligadas a la búsqueda, uso y evaluación 
de la información, incluyendo las Tecnologías de la Información y la Comunicación como medio para ello.

Entendiendo que las dos estrategias anteriormente referidas son propuestas parciales, se opta por utilizar como punto de partida la iniciativa el proyecto de Marco Común de Competencia Digital Docente, diseñado por el Instituto Nacional de Tecnologías Educativas y de Formación del Profesorado en 2013. Esta propuesta nace con la intención de ofrecer una referencia descriptiva que pueda servir con fines de formación y en procesos de evaluación y acreditación. Forma parte tanto del Plan de Cultura Digital en la Escuela (2013) como del Marco Estratégico de Desarrollo Profesional Docente (2013) y fue elaborado a partir del Proyecto DIGCOMP: A framework for developing and undersanding digital competence in Europe de la Unión Europea (Ferrari, 2013).

De este modo, los objetivos de este marco serían, entre otros, facilitar una referencia común a profesores y formadores, contribuir a una mayor exigencia en relación a la competencia digital docente o disponer de una lista estatal de competencias mínimas para los profesores. Este marco establece cinco áreas de competencia digital (Información, Comunicación, Creación de Contenido, Seguridad y Resolución de problemas), que serán explicadas con mayor detalle en el siguiente punto.

Cabe exponer, por último, que el sujeto objeto de estudio de este trabajo es el estudiante, y se considera como una variable interviniente en los resultados el hecho de que estos futuros maestros se forman en un entorno de aprendizaje virtual donde las características del escenario de aprendizaje mejoran las habilidades en el uso de TIC (HernándezCarranza, Romero-Corella y Ramírez-Montoya, 2015).

\section{Estudios y experiencias sobre competencia digital en otros contextos}

Como se ha expuesto, existe un marco normativo que ha favorecido una integración progresiva de las Tecnologías de la Información y la Comunicación en los sistemas educativos.

Igualmente, se ha constatado que la formación del profesorado en esta área ha sido una preocupación constante de las Administraciones, que ha llevado a trabajar en marcos que delimitaran los aspectos fundamentales que debían componer la capacitación tecnológica de los docentes. 
Ahora bien, cabría preguntarse: ¿realmente hay una necesidad de formarse en materia digital cuando a diario se manejan las tecnologías en los diferentes ámbitos de la vida? ¿Cuál es el nivel de competencia digital de la sociedad y el profesorado de nuestro país?

Según el We Are Social (2016), España es el país de Europa con una mayor tasa de penetración de las redes sociales, siendo el dato de un $47 \%$ frente al $29 \%$ global. Esta cifra permite comprobar que hay un uso extendido de las TIC que no necesariamente implica una competencia digital que sustente un buen uso de las mismas.

De hecho, según Ferrés, Aguaded y García-Matilla (2012), los españoles suspenden en materia de competencia multimedia e informacional, y únicamente alcanzan un nivel competencial suficiente -del $61 \%$ - debido a la consecución de habilidades técnicas e instrumentales. En opinión de Ferrés, Aguaded y García-Matilla (2012) "los resultados de esta investigación deberían constituir un reto para las instancias educativas que han de comprometerse en garantizar una formación que potencie la competencia mediática de la ciudadanía" (p.24).

Centrando el análisis en la competencia docente, Prendes y Gutiérrez (2011) identifican la competencia tecnológica como aquella relacionada con "la capacidad, conocimiento y actitud de uso de las tecnologías de la información y la comunicación en sus diversas funciones y contextos de aplicación" (p.2). Y así lo hacen para, a continuación, plantear algunos de los estándares internacionales que se han empleado, hasta la fecha, en el análisis de dichas competencias TIC (Tecnologías de la Información y la Comunicación). De este modo señalan que, en Europa, la entrada de las competencias en el ámbito universitario se ha Ilevado a cabo con el proyecto Tunning del que derivarían marcos referenciales como el proyecto «Estándares de competencias TIC para docentes de la UNESCO», elaborado en 2008 y revisado en 2011. Asimismo, tras su análisis de las competencias del profesorado en las universidades españolas sentencian que el $91 \%$ de los docentes estudiados demuestra un elevado conocimiento general sobre las posibilidades de las TIC.

En esta misma línea, Prendes y Gutiérrez (2013) realizaron un análisis de las competencias tecnológicas del profesorado en las Universidades españolas, concluyendo que había una situación diversa, tanto en las competencias asociadas a la gestión, como a la docencia y a la investigación.

Por último, hay dos estudios similares que han abordado la percep- 
ción de los estudiantes con respecto a su competencia digital. Gutiérrez y Serrano (2016) concluyen que los estudiantes del Grado en Educación Primaria de la Universidad de Murcia tienen una percepción elevada de su nivel de competencia digital docente en aspectos básicos, mostrando mayores dificultades en las áreas de creación de contenidos y seguridad. Igualmente, en el trabajo de Cabezas, Casillas y Pinto (2014), realizado con estudiantes de la Universidad de Salamanca, se concluye que los estudiantes se sienten capacitados para implementar las Tecnologías en los procesos de enseñanza y aprendizaje.

Teniendo en cuenta todo lo expuesto, y partiendo de las áreas y criterios que se recogen en el Marco Común de Competencia Digital Docente, se establecen como objetivos del presente trabajo los siguientes:

1. Analizar y describir la percepción de los estudiantes de los Grados en Educación Infantil y Primaria con respecto a su competencia digital docente.

2. Observar correlaciones entre el nivel de competencia digital y variables de índole personal (como la edad) y académicas (como el curso en que está matriculado o la mención cursada).

3. Analizar las diferencias entre la percepción de los estudiantes con respecto a su competencia digital y los resultados que se desprenden del cuestionario.

\section{Metodología}

La población a la que se dirigía este estudio estaba conformada por los estudiantes matriculados en el Grado de Educación Infantil y el Grado en Educación Primaria en el curso 2014-2015 (651 estudiantes) de la Universidad Internacional de Valencia (VIU).

Finalmente, la muestra está compuesta por 104 estudiantes de estas titulaciones $(n=104)$, un $16 \%$ de la población de referencia.

En cuanto a las características de esta muestra, está constituida mayoritariamente por mujeres (90.4\%), de nacionalidad española (93.3\%), de edades comprendidas entre los 31 y los 35 años (30.8\%), que en el $57.7 \%$ de los casos habían cursado estudios superiores previamente. El $60.6 \%$ de los estudiantes que han cumplimentado el cuestionario procede del título universitario de Educación Primaria mientras que el restante $39.4 \%$ accede desde el grado de Educación Infantil. Analizados 
conjuntamente, el $37.5 \%$ de la muestra lo conforman estudiantes de cuarto curso (seguidos de los de primer curso) y la especialidad más representada (24.0\%) es la mención en Tecnologías de la Información y la Comunicación.

Acorde con los objetivos de este trabajo, el diseño de investigación seleccionado es cuantitativo y descriptivo (no experimental). En este sentido, nos aproximamos al objeto de estudio sin modificar las variables, con la finalidad de explorarlo y describirlo.

La recogida de datos se ha llevado a cabo a través de un cuestionario diseñado mediante Google Forms. Dicho instrumento ha sido diseñado ad-hoc para esta investigación, estando compuesto de dos partes diferenciadas: una parte orientada a conocer algunas características personales del futuro maestro que cumplimentaba el instrumento y que recogía información de variables como el sexo (hombre/mujer), año de nacimiento, país de origen, titulación previa, curso y mención del Grado de la que se ha matriculado. Además, se añadía, un ítem final en el que los estudiantes debían manifestar la percepción sobre su nivel de competencia digital (inicial o básico, intermedio o usuario y avanzado), que nos permitirá, como se verá más adelante, confrontar la propia percepción con las puntuaciones que se derivan de la encuesta.

La segunda parte de la encuesta se compone de 21 ítems, que se asocian a los 21 criterios que componen el Marco Común de la Competencia Digital Docente, divididos en las áreas expuestas anteriormente (Información, Comunicación, Creación de Contenidos, Seguridad y Resolución de Problemas).

En este sentido, se crea un instrumento específico para esta investigación porque, aunque la segunda parte reproduce de forma ajustada las dimensiones y escalas competenciales que contempla el documento oficial del DIGCOMP, su propuesta no propone la recogida de datos sociodemográficos que permitan establecer correlaciones.

Cada una de estas áreas, incluye las siguientes competencias: 


\section{Tabla 1. Áreas y competencias del Marco Común de Competencia Digital Docente.}

\begin{tabular}{ll}
\hline Áreas & Competencias \\
\hline 1. Información & 1.1. Navegación, búsqueda y filtrado de información. \\
& 1.2. Evaluación de información. \\
& 1.3. Almacenamiento y recuperación de información \\
\hline 2. Comunicación & 2.1. Interacción mediante nuevas tecnologías. \\
& 2.2. Compartir información y contenidos. \\
& 2.3. Participación ciudadana en línea. \\
& 2.4. Colaboración mediante canales digitales. \\
& 2.5. Netiqueta. \\
2.6. Gestión de la identidad digital
\end{tabular}

El modo de responder a la encuesta consistía en la elección, por parte del estudiante de la afirmación de las tres disponibles que más se aproximara a su destreza en cada uno de los criterios.

Cada una de las respuestas se asocia a un nivel de competencia diferente, habiendo tres niveles: A- Inicial o Básico; B- Intermedio y C- Avanzado.

Estos niveles nos permiten, finalmente, puntuar y evaluar la competencia digital de los estudiantes, asociando a cada uno de ellos un valor numérico.

Además, para poder analizar la competencia de los estudiantes de un modo global, se han delimitado tres niveles en función de la puntuación obtenida en las respuestas a los 21 ítems analizados (con un rango entre 21 y 63 puntos).

Se realiza un análisis descriptivo e interpretativo de los datos, habiéndose utilizado para este análisis el paquete estadístico SPSS v.20 y Microsoft Excel 2010. 


\section{Resultados}

En este apartado se exponen los resultados que se derivan del análisis cuantitativo de las respuestas aportadas por los participantes.

Se presentará, en primer lugar, un análisis global de los resultados por dimensiones, atendiendo a las puntuaciones obtenidas en las áreas de Información, Comunicación, Creación de Contenidos, Seguridad y Resolución de Problemas.

En segundo lugar, se recogerán los resultados del análisis de los resultados en función de algunas variables como la edad, el curso en que están matriculados los estudiantes o la mención cursada.

En último lugar, se expondrá la contraposición entre el nivel de competencia digital percibido y el que se deriva de los resultados del cuestionario.

\section{Análisis dimensional de los resultados}

Tal y como se recogía en la Metodología, para explorar de un modo global las competencias en las áreas que forman parte del Marco Común de Competencia Digital Docente, se ha establecido un sistema de puntuaciones en una escala de 21 a 63 puntos que nos permite analizar un nivel de competencia general. Posteriormente, se han codificado estos puntos en los tres niveles que se recogen en el documento (1: básico, 2: intermedio, 3: avanzado).

Este paso nos permite ver que las puntuaciones medias varían entre 1.83 y 2.45 en función del área de competencia.

El área donde los estudiantes tienen una media mayor (2.45 sobre 3), aproximándose a un nivel de competencia avanzado, sería el área de la Información, que se vincula a la capacidad para identificar, localizar, recuperar, almacenar, organizar y analizar la información digital, evaluando su finalidad y relevancia.

El nivel de competencia sería intermedio en las áreas de Comunicación (2.24) y Seguridad (2.08). El área de Comunicación implica la capacidad para comunicar en entornos digitales, compartir recursos a través de herramientas en línea, conectar y colaborar con otros a través de herramientas digitales, interactuar y participar en comunidades y redes. Mientras, el área de Seguridad, se asocia a aspectos como la protección personal, protección de datos, protección de la identidad digital, uso de seguridad, y el uso seguro y sostenible de las TIC. 
En el caso de las otras dos áreas, las puntuaciones se sitúan por debajo del 2. Concretamente, la puntuación del área relacionada con la Resolución de problemas sería de 1.94. Esta área se asocia a la competencia del estudiante para identificar necesidades y recursos digitales, tomar decisiones a la hora de elegir la herramienta digital apropiada, acorde a la finalidad o necesidad, resolver problemas conceptuales a través de medios digitales, resolver problemas técnicos, el uso creativo de la tecnología, y actualizar la competencia propia y la de otros.

Por último, en el área de Creación de contenidos, la puntuación es de 1.83, que se asocia a capacidades para desarrollar tareas como crear y editar contenidos nuevos (textos, imágenes, videos...), integrar y reelaborar conocimientos y contenidos previos, realizar producciones artísticas, contenidos multimedia y programación informática, saber aplicar los derechos de propiedad intelectual y las licencias de uso.

Estas puntuaciones medias responden a una medición global de la competencia digital por áreas, que puede verse de un modo más desglosado en la figura 1 , donde se recoge el porcentaje de estudiantes en cada uno de los niveles por área.

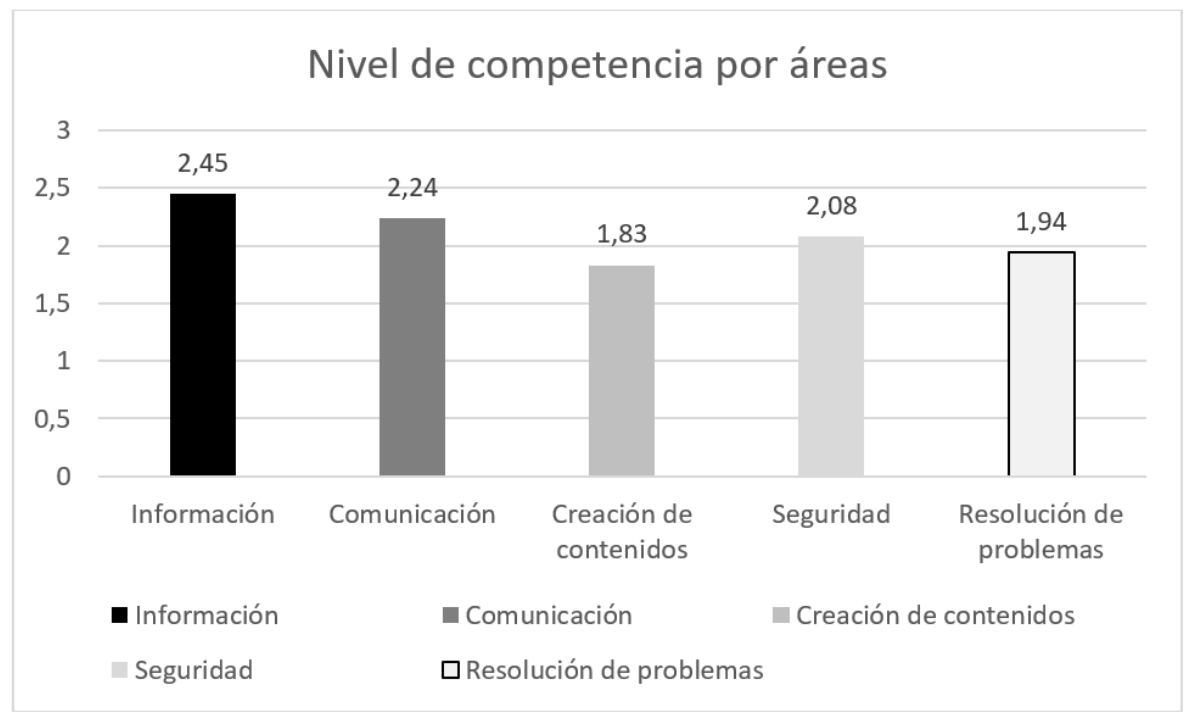

Figura 1. Puntuaciones medias por área. 


\section{Análisis competencial por variables}

Las características de los estudiantes son diversas, por lo que resulta interesante analizar si existe algún tipo de correlación entre algunas variables de índole personal y el nivel de competencia digital.

No se realiza un estudio de la variable sexo, puesto que las muestras no son equiparables (un $90.4 \%$ de la muestra son mujeres y un $9.6 \%$ de la muestra son hombres).

Para analizar si existe alguna relación entre la edad y la competencia digital, se ha dividido a la muestra en dos segmentos. Partiendo de que las edades están comprendidas entre los 20 y los 49 años, quedando estructurada la muestra en los siguientes grupos:

- Estudiantes de entre 20 y 35 años: 61 personas comprenden este grupo.

- Estudiantes de entre 36 y 49 años: este grupo está compuesto por 43 personas.

Si se atiende a la competencia digital de estos dos grupos, los más jóvenes (20-35 años), tienen 44.66 puntos de media sobre los 66 puntos posibles. Por otro lado, el grupo de los sujetos comprendidos entre los 36 y los 49 años tendrían una puntuación media de 43.38.

Analizando si existen diferencias en el nivel de competencia digital en función del curso en que se está matriculado, puede observarse que la puntuación media va creciendo según se avanza en el plan de estudios.

La puntuación media de los estudiantes de primer curso sería de 42.03 puntos, de 43.40 puntos en segundo, de 44.77 puntos en tercero y de 46.15 puntos en cuarto. 


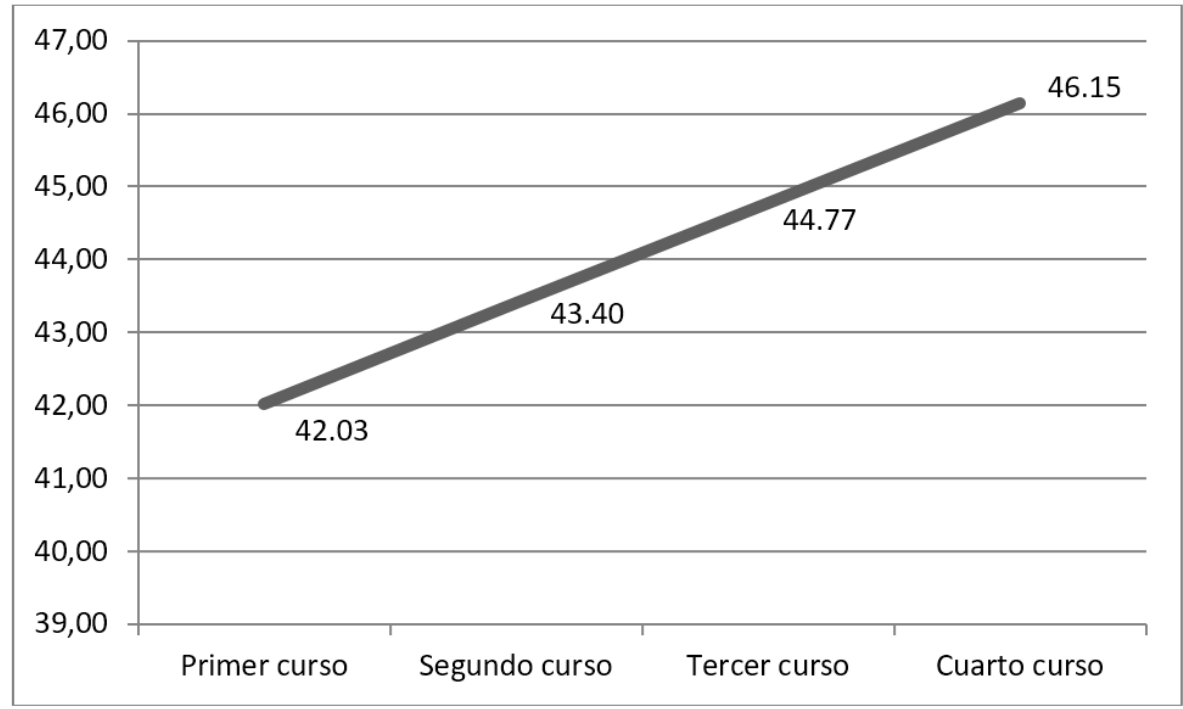

Figura 2. Puntuación media de competencia digital por curso.

Por último, si se explora el nivel de competencia digital en función de la mención cursada (Lengua Extranjera: Inglés, Lengua Valenciana y Tecnologías de la Información y la Comunicación), se deduce que los estudiantes de la mención en TIC obtendrían una puntuación media de 48.82 puntos, teniendo una puntuación media de 43.71 puntos los estudiantes de la mención de Lengua Valenciana y 40.38 los de Lengua Extranjera: Inglés.

\section{Análisis de la competencia real frente a la competencia percibida}

Por último, es interesante analizar si los estudiantes tienen una percepción ajustada de su propia competencia digital docente.

En este sentido, se incluía un ítem en el cuestionario donde se les solicitaba que expusieran cuál pensaban que era su nivel de competencia digital docente (Inicial o básico, Intermedio o usuario o Avanzado).

Para contraponer esta percepción con la competencia real, se han sumado las puntuaciones de los estudiantes en cada uno de los ítems que componen la encuesta (21 ítems), otorgando un punto cuando la respuesta se asociaba a un nivel de competencia básico, dos puntos cuando se asociaba a un nivel de competencia intermedio y tres cuando se asociaba a un nivel de competencia avanzado. 
De este modo, y considerando que todas las respuestas quedarían enmarcadas en un rango de entre 21 y 63 puntos, se han establecido los tres niveles competenciales (Básico, Intermedio y Avanzado).

Siguiendo esta escala, habría 18 estudiantes (un 17.3\%) con un nivel de competencia básico, 58 estudiantes (55.8\%) con un nivel intermedio y $28(26.9 \%)$ con un nivel de competencia avanzado.

Sin embargo, si se atiende a la percepción de los estudiantes sobre su propia competencia digital, 3 de ellos consideran tener un nivel de competencia básico (2.9\%), 69 un nivel de competencia intermedio $(66.3 \%)$ y 31 de ellos (19.8\%) un nivel de competencia avanzado.

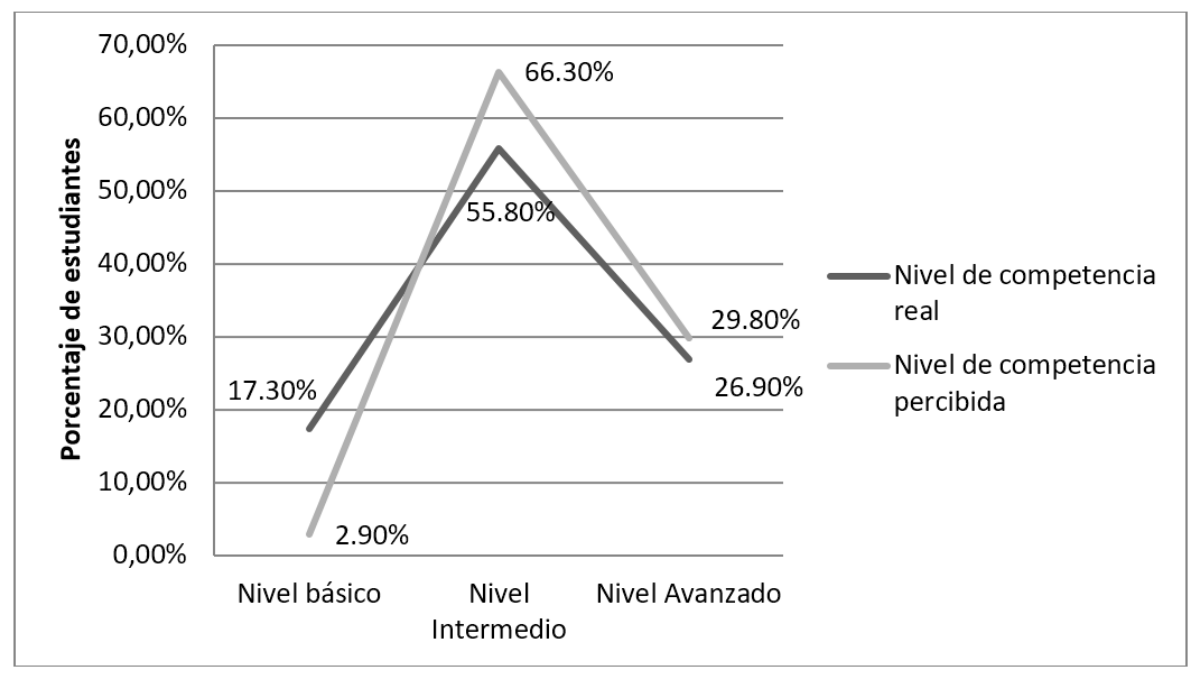

Figura 3. Nivel de competencia real frene a nivel de competencia percibida.

Si se concretan los datos, y atendiendo caso por caso a si tienen una percepción acertada de sus propias competencias, se encuentra que:

- 24 estudiantes (un 23.1\%) tienen la percepción de que su nivel de competencia es superior a lo que realmente sería

- 6 de los estudiantes (un 5.8\%) pensarían que su nivel es inferior a lo que realmente es.

- La gran mayoría (74 estudiantes, que representan un $71.2 \%$ del total) tendrían una percepción acertada de sus competencias. 
Vicente Gabarda Méndez, Ana Rodríguez Martín y María Dolores Moreno Rodríguez

\section{Conclusiones}

El Marco de Competencia Digital Docente (INTEF, 2013) ofrece un escenario donde poder identificar y evaluar las competencias y destrezas que cualquier profesional docente debe adquirir y desarrollar para poder facilitar el desarrollo de la competencia digital docente en sus estudiantes.

Tomando como referencia este estándar, y dando respuesta al objetivo 1,puede concluirse que los estudiantes de los títulos de Magisterio de la Universidad VIU tienen un nivel de competencia intermedio, obteniendo, como dato global, un 2,1 sobre 3 .

Los mayores niveles de competencia del alumnado se asocian al área de la Información (2.45 sobre 3). En esta área, los estudiantes tienen mayoritariamente un nivel de competencia avanzado en Búsqueda de la Información (53.9\%) y Evaluación de la Información (66.4\%).

Coinciden estos datos con la tesis de Ferrés, Aguaded y García-MatiIla (2012) en que existe una competencia suficiente en el área informacional.

Por el lado contrario, las mayores carencias se observan en el área de Creación de Contenidos (1.83 puntos sobre 3), especialmente en el conocimiento y aplicación de los Derechos de Autor (un 63.5\% de los estudiantes tienen un nivel de competencia básico) y en Programación, donde un $76 \%$ de ellos también tiene este nivel de competencia.

Si se contraponen estos datos con las conclusiones que obtuvieron Prendes y Gutiérrez (2011), donde había un altísimo porcentaje de docentes con un elevado conocimiento general de las TIC, se observa que este conocimiento puede no darse en todas las áreas por igual, coincidiendo así con la propuesta de las mismas autoras en 2013.

Resultados similares obtuvieron Angulo et al. (2015) y Roblizo y Cózar (2015) donde se concluía que los docentes tenían altos niveles de competencia en cuestiones básicas y carencias específicas en aquellas destrezas que requerían algo más de especialización.

Estas carencias, especialmente en algunas áreas, puede ser la causa de una realidad constatada por Fernández-Cruz y Fernández-Díaz (2016), un estudio en el que concluían que había una contradicción evidente entre las competencias que debe tener un docente para desarrollar la competencia digital de sus estudiantes y las que realmente poseen.

Atendiendo al cruce de la competencia digital con variables personales y académicas (objetivo 2), como puede observarse no existe una 
correlación significativa entre la edad y la competencia digital. Este dato difiere, por ejemplo, de las encuestas que miden, de forma global, el uso de las tecnologías en el hogar (Instituto Nacional de Estadística, 2014), donde hay un decrecimiento importante de su utilización según avanza la edad.

Por otra parte, se partía de la tesis de que el nivel de competencia digital de los estudiantes sería mayor en estudiantes de último curso que en los de primero. Esta creencia parte de la idea de que, al estar la metodología y los procesos de enseñanza y aprendizaje mediado por las TIC, los estudiantes podrían tener una mayor competencia digital según avanzan en el plan de estudios. En este caso, sí puede observarse una correlación positiva entre el curso y la puntuación media, constatando la tesis de Hernández-Carranza, Romero-Corella y Ramírez-Montoya (2015) sobre la importancia de formarse en un escenario virtual para la mejora de las habilidades en el uso de TIC.

Se partía también de la percepción de que, dado que entre las menciones que se ofertan en las titulaciones analizadas se encuentra una especialización en Tecnologías de la Información y la Comunicación, donde se abordan contenidos y competencias específicas ligadas a las tecnologías, los estudiantes matriculados en ella podrían tener un mayor nivel de competencia digital que los que cursan otras especialidades. En este caso, aunque sí hay diferencias entre las puntuaciones de los estudiantes de esta mención y las del resto de menciones, la variación no es significativa, cuestión que nos lleva a reflexionar acerca de las competencias que deberían desarrollarse, con éxito relativo, en la mención de Tecnologías de la Información y la Comunicación.

Por otra parte, tal y como ha podido constatarse, hay una diferencia importante entre el nivel de competencia digital de los estudiantes y la percepción que tienen sobre dicha competencia (objetivo 3). Esta discrepancia es especialmente destacable en el nivel de competencia básico, donde encajarían un total de 18 estudiantes y solamente tres consideran tenerlo. Se concluye, por tanto, que los estudiantes de los Grados de Magisterio tienen una percepción de que su nivel de competencia digital es mayor de lo que realmente sería.

Se finaliza apuntando que, tal y como se ha podido constatar, existe una incongruencia entre la importancia que se le concede a las TIC y a la competencia digital en la normativa (tanto en la LOMCE como en los Reales Decretos de cada una de las etapas) y el reflejo real que tiene 
sobre la implementación de políticas de formación del profesorado en la realidad.

Capilla, Torres y Sánchez (2015) aludían a que la puesta en marcha del Espacio Europeo de Educación superior podía suponer un punto de partida importante para la integración de las TIC en los sistemas educativos, aunque Herrada y Herrada (2011) apuntaban que, con la implantación del EEES, habían decrecido los contenidos TIC en las titulaciones de magisterio.

Esta misma línea puede verse en la implementación del propio Marco de Competencia Digital Docente, constatándose una falta de vinculación entre el documento y su aplicación práctica en las escuelas (López y Catasús, 2015).

Por último, contrastando los resultados del presente trabajo con estudios precedentes enmarcados en la misma línea, Gewerc y Montero (2015) concluían que sus futuros docentes poseen una perspectiva eminentemente instrumental de las tecnologías y Gutiérrez y Serrano (2016) exponían que el nivel de competencia mayor se asociaba a las áreas de información y comunicación.

En el caso de nuestro estudio, se confirma esta tendencia, coincidiendo en que los estudiantes de magisterio de la Universidad VIU muestran un nivel de competencia mayor en estas dos áreas, que podría considerarse que albergan destrezas más básicas.

Asimismo, la percepción positiva que tienen nuestros estudiantes sobre su competencia digital va en la línea de las conclusiones de estudios como el de Cabezas, Casillas y Pinto (2014).

Se concluye apuntando que la necesidad de implementar las TIC como parte esencial de la formación de profesores y estudiantes no solamente es una intención política y normativa, sino que es un hecho constatado por parte de los diferentes agentes como concluyen los estudios de Capilla, Torres y Sánchez (2015) y Morales, Trujillo y Raso (2015).

Habiendo respondido a los tres objetivos que se planteaban en este trabajo, se plantean algunas limitaciones que deberían ser tomadas en cuenta para futuros estudios. Aunque se ha podido replicar el trabajo con otras investigaciones similares en el contexto universitario, sería recomendable seguir contrastando los resultados con futuras investigaciones que se fundamenten en el Marco Común de Competencia Digital Docente. Asimismo, con el objetivo de seguir mejorando la práctica educativa, se está trabajando en el análisis de la percepción sobre la competencia digital del futuro profesorado de Secundaria. 
La competencia digital en estudiantes de magisterio. Análisis competencial y percepción personal del futuro maestro

Vicente Gabarda Méndez, Ana Rodríguez Martín y María Dolores Moreno Rodríguez

\section{Referencias bibliográficas}

Angulo, J., García-López, R.I, Torres, C.A., Pizá, R.I y Rodrigo, E. (2015). Nivel de Logro de Competencias Tecnológicas del Profesorado Universitario. International Multilingual Journal of Contemporary Research, 3 (1), 67-80. doi: http://dx.doi.org/10.15640/ imjcr.v3n1a8. Recuperado de http:// http://imjcr.com/journals/imjcr/Vol_3_No_1_ June_2014/8.pdf

Cabezas, M., Casillas, S. y Pinto, A.M. (2014). Percepción de los alumnos de Educación Primaria de la universidad de Salamanca sobre su competencia digital. EDUTEC. Revista Electrónica de Tecnología Educativa, $n^{\circ}$ 48, 1-14. Recuperado de http://www. edutec.es/revista/index.php/edutec-e/article/view/156/21.

Capilla, M. M., Torres, J. M. T., \& Sánchez, F. R. (2015). Percepciones acerca de la integración de las TIC en el proceso de enseñanza-aprendizaje de la universidad. Pixe/Bit: Revista de medios y educación, $n^{\circ} 46,103-117$. doi: http://dx.doi.org/10.12795/ pixelbit.2015.i46.07. Recuperado de http://acdc.sav.us.es/pixelbit/images/stories/ p46/07.pdf

Comunidades Europeas (2006). Recomendación 2006/962/CE del Parlamento Europeo y del Consejo, de 18 de diciembre de 2006, sobre las competencias clave para el aprendizaje permanente. Diario Oficial L 394 de 30.12.2006. Recuperado de http:// eur-lex.europa.eu/legal-content/ES/TXT/?uri=celex\%3A32006H0962

Fernández-Cruz, F. J., \& Fernández-Díaz, M. J. (2016). Los docentes de la generación Z y sus competencias digitales. Comunicar. Revista Científica de Comunicación y Educación, no 46, 97-105. doi: http://dx.doi.org/10.3916/C46-2016-10. Recuperado de http://www.revistacomunicar.com/index.php?contenido=detalles\&numero $=46 \& \mathrm{a}$ rticulo=46-2016-10

Ferrari, A. (2013). DIGCOMP: A Framework for Developing and Understanding Digital Competence in Europe. Recuperado de http://ipts.jrc.ec.europa.eu/publications/pub. $\mathrm{cfm}$ ?id=6359

Ferrés, J., Aguaded, I., \& García-Matilla, A. (2012). La competencia mediática de la ciudadanía española: dificultades y retos. Revista ICONO14. Revista Científica De Comunicación y Tecnologías Emergentes, 10 (3), 23-42.doi:http://dx.doi.org/10.7195/ ri14.v10i3.201. Recuperado de http://www.icono14.net/ojs/index.php/icono14/article/view/201

Gewerc, A., \& Montero, L. (2015). Conocimiento profesional y competencia digital en la formación del profesorado. El caso del Grado de Maestro en Educación Primaria. Revista Latinoamericana de Tecnología Educativa-RELATEC, 14 (1), 31-43. (http://goo. gl/XKFzzj) (5-2-2016).

Gutiérrez y Soriano (2016) Evaluación y desarrollo de la competencia digital de futuros maestros en la Universidad de Murcia. Journal of new Approaches in Educational Research. 5 (1), 53-59. doi: 10.7821/naer.2016.1.152. Recuperado de http://naerjournal.ua.es/article/view/v5n1-8.

Hernández-Carranza, E. E., Romero-Corella, S. I., y Ramírez-Montoya, M. S. (2015). Evaluación de competencias digitales didácticas en cursos masivos abiertos: Contribución al movimiento latinoamericano. Comunicar: Revista Científica de Comu- 
La competencia digital en estudiantes de magisterio. Análisis competencial y percepción personal del futuro maestro

Vicente Gabarda Méndez, Ana Rodríguez Martín y María Dolores Moreno Rodríguez

nicación y Educación, 22 (44) , 81-90. doi: http://dx.doi.org/10.3916/C44-2015-09. Recuperado de http://www.revistacomunicar.com/verpdf.php?numero=44\&articu $\mathrm{lo}=44-2015-09$

Herrada, R.I. y Herrada, G. (2011). Adaptación de los estudios de Magisterio al EEES: las TIC en los nuevos planes de estudio. Edutec-e. Revista Electrónica de Tecnnología Educativa 36. pp. 1-12. Recuperado de: http://www.edutec.es/revista/index.php/ edutec-e/article/view/405/141

International Society for Technology in Education (2008). The ISTE NETS and Performance Indicators for Teachers. Recuperado de http://www.iste.org/standards/ISTEstandards/standards-for-teachers

Instituto Nacional de Estadística (2014). Encuesta sobre Equipamiento y Uso de Tecnologías de Información y Comunicación en los hogares 2014. Recuperado de http:// www.ine.es/dynt3/inebase/index.htm?type=pcaxis\&path=/t25/p450/base_2011/ a2014/\&file=pcaxis

Instituto Nacional de Tecnologías Educativas y de Formación del Profesorado (2014). Marco común de competencia digital docente. Recuperado de http://educalab.es/ documents/10180/12809/MarcoComunCompeDigiDoceV2.pdf

López, G. D., y Catasús, M. G. (2015). La competencia digital en la enseñanza del diseño. El caso de BAU Centro Universitario de Diseño de Barcelona (UVic). Revista Latinoamericana de Tecnología Educativa-RELATEC, 14 (2), 51-65. Recuperado de http://relatec.unex.es/article/view/1623

Ministerio de Educación y Ciencia (1990). Ley Orgánica 1/1990, de 4 de octubre, de Ordenación General del Sistema Educativo. Boletín Oficial del Estado, n² 238. Recuperado de https://www.boe.es/buscar/doc.php?id=BOE-A-1990-24172

Ministerio de Educación, Cultura y Deporte (2002). Ley Orgánica 10/2002, de 23 de diciembre, de Calidad de la Educación. Boletín Oficial del Estado, n 307. Recuperado de https://www.boe.es/buscar/doc.php?id=BOE-A-2002-25037

Ministerio de Educación, Cultura y Deporte (2006). Ley Orgánica 2/2006, de 3 de mayo, de Educación. Boletín Oficial del Estado, no 106. Recuperado de https://www.boe.es/ buscar/act.php?id=BOE-A-2006-7899

Ministerio de Educación y Ciencia (2007). Real Decreto 1630/2006, de 29 de diciembre, por el que se establecen las enseñanzas mínimas del segundo ciclo de Educación infantil. BOE $\mathrm{n}^{\circ} 4$. Recuperado en https://www.boe.es/diario_boe/txt.php?id=BOEA-2007-185

Ministerio de Educación y Ciencia (2007). Orden ECI/3854/2007, de 27 de diciembre, por la que se establecen los requisitos para la verificación de los títulos universitarios oficiales que habiliten para el ejercicio de la profesión de Maestro en Educación Infantil. BOE no 312. Recuperado de https://www.boe.es/diario_boe/txt.php?id=BOEA-2007-22446

Ministerio de Educación y Ciencia (2007). Orden ECI/3857/2007, de 27 de diciembre, por la que se establecen los requisitos para la verificación de los títulos universitarios oficiales que habiliten para el ejercicio de la profesión de Maestro en Educación Primaria. BOE ${ }^{\circ}$ 312. Recuperado de https://www.boe.es/diario_boe/txt.php?id=BOEA-2007-22449 
La competencia digital en estudiantes de magisterio. Análisis competencial y percepción personal del futuro maestro

Vicente Gabarda Méndez, Ana Rodríguez Martín y María Dolores Moreno Rodríguez

Ministerio de Educación, Cultura y Deporte (2013). Ley Orgánica 8/2013, de 9 de diciembre, para la Mejora de la Calidad Educativa. Boletín Oficial del Estado, no 295. Recuperado de https://www.boe.es/boe/dias/2013/12/10/pdfs/BOE-A-2013-12886. pdf

Ministerio de Educación, Cultura y Deporte (2014). Real Decreto 126/2014, de 28 de febrero, por el que se establece el currículo básico de la Educación Primaria. BOE, n 52. Recuperado en https://www.boe.es/buscar/doc.php?id=BOE-A-2014-2222

Morales, M., Trujillo, J. M. y Raso, F. (2015). Percepciones acerca de la integración de las TIC en el proceso de enseñanza-aprendizaje de la Universidad. Píxel-Bit. Revista de Medios y Educación. 46, 103-117. doi: http://dx.doi.org/10.12795/pixelbit.2015. i46.07. Recuperado de http://acdc.sav.us.es/pixelbit/images/stories/p46/07.pdf

Organización de las Naciones Unidas para la Educación, la Ciencia y la Cultura (2014). Orientación normativa y estratégica de la alfabetización mediática e informacional. Recuperado en http://unesdoc.unesco.org/images/0021/002160/216099S.pdf

Prendes, M. P. y Gutiérrez, I. (2011). Soportes tecnológicos para la enseñanza y el aprendizaje In: Cebrián de la Serna, M. Procesos educativos con TIC en la sociedad del conocimiento (pp. 163-178). Madrid: Pirámide.

Prendes, M. P. y Gutiérrez, I. (2013). Competencias tecnológicas del profesorado en las universidades españolas. Revista de Educación, 361, 196-222. doi 10.4438/1988592X-RE-2011-361-140. Recuperado en http://www.revistaeducacion.mec.es/ doi/361_140.pdf

Roblizo, M. J., y Cózar, R. (2015). Usos y competencias en TIC en los futuros maestros de educación infantil y primaria: Hacia una alfabetización tecnológica real para docentes. Pixel-Bit: Revista de medios y educación, 47. 23-39. doi: http://dx.doi. org/10.12795/pixelbit.2015.i47.02. Recuperado en http://acdc.sav.us.es/pixelbit/ images/stories/p47/02.pdf

We Are Social (2016). Estudio sobre el estado de Internet y las Redes Sociales en España y en todo el mundo. Recuperado en http://wearesocial.com/uk/special-reports/ digital-in-2016 